Authors Postprint of:

Amadi HO, Hansen UN, Wallace AL, Bull AMJ. A scapular coordinate frame for clinical and kinematic analyses. J Biomech 41: (2008), 2144-2149.

Publishers version can be found here: http://www.sciencedirect.com/science/journal/00219290

\title{
A scapular coordinate frame for clinical and kinematic analyses
}

\author{
Authors: $\quad$ Hippolite O Amadi $\mathrm{PhD}^{1,2}$, Ulrich N Hansen $\mathrm{PhD}^{2}$, Andrew L Wallace PhD FRACS ${ }^{3,4}$, Anthony MJ Bull \\ $\mathrm{PhD}^{1}$ \\ ${ }^{1}$ Department of Bioengineering, imperial College London, UK \\ ${ }^{2}$ Department of Mechanical Engineering, Imperial College London \\ ${ }^{3}$ Musculoskeletal Surgery, Imperial College London, UK \\ ${ }^{4}$ Shoulder Unit, Hospital of St John and St Elizabeth, London, UK
}

Corresponding Author:

Dr Anthony MJ Bull
Department of Bioengineering
Imperial College London
South Kensington Campus
London SW7 2AZ,
United Kingdom
+44207594 5186
a.bull@imperial.ac.uk

Keywords: $\quad$ Scapula, coordinate, kinematics, morphometry

\begin{abstract}
The aim of this study was to define a body-fixed coordinate frame for the scapula that minimises axes variability and is closely related to the clinical frame of reference. Medical images of twenty-one scapulae were used to quantify fourteen different axes from identifiable landmarks. The plane of the blade of the scapula was defined. The orientations of the quantified axes to this were calculated. The angular relationships between axes were quantified and applied to grade the sensitivity of each axis to inter-scapular variations in the others. The volume of data required to define an axis was noted for its dependency on pathology and the three criteria were weighted according to relative importance. The two axes with the highest weighting were applied to define a body-fixed Cartesian coordinate frame for the scapula. A least square medio-lateral line through the centre of the spine root was the most optimal axis. The plane formed by the spine root line and a least square line through the centre of the lateral border ridge was the most optimal scapular plane. This body-fixed Cartesian coordinate frame is closely aligned to the cardinal planes in the anatomical position and thus is a clinically-applicable, specimen invariant coordinate frame that can be used in patient-specific kinematics modelling.
\end{abstract}

\section{Introduction}

It is known that the functional kinematics of the glenohumeral joint is a function of the coordinated operations of the soft tissues (Lembeck and Wülker, 2004; McMahon et al., 2004; Wong et al., 2003) and the characteristic geometry of the associated bones (Hess, 2000; Lehtinen et al., 2004), including scapular glenoid morphology (Hess, 2000; Lehtinen et al., 2004; Wong et al., 2003). Different coordinate frames have been proposed for defining and describing the motion of the scapula in space and the relative motion of the scapula and humerus (Hill, 2006; Johnson et al., 1993; Novotny et al., 2000; Van der Helm, 1997). The choice of anatomical landmarks for the definition of some of these frames results in principal axes that are oblique to the cardinal planes (coronal, sagittal, axial) in the standard anatomical position in which a person is upright and erect on both legs, facing forwards, with the arms at the sides and the palms turned forwards. This results in difficulty in relating data presented using these frames to the clinical situation. Therefore there is a need to design a clinically compatible coordinate frame that will be able to relate to anatomical cardinal directions. In addition, the morphology of the scapula is highly variable between individuals (Gallino et al., 1998; Stehle et al., 2007). Therefore there is a need to define anatomical scapular landmarks that are less variable between individuals than the variability of the overall shape. Also, some of the landmarks used in the literature are highly dependent on the presence of pathology, thus introducing more variability in the coordinate frame. Therefore, a pathology-insensitive approach to defining the scapular coordinate frame must be developed.

There are numerous methods that are commonly used in the description and study of shoulder kinematics (Hill et al., 2007; Novotny et al., 2000; Van der Helm, 1994; Van der Helm and Pronk, 1995; Wu et al, 2005). The description and use of a particular coordinate system among researchers have so far depended on the individual's choice and convenience as it affected the particular study at hand. All of these methods have used landmarks on the surface of the scapula to establish a repeatable coordinate frame in space.

Definition of functional coordinate frames for individual scapulae using subjective combinations of these axes is susceptible to inconsistencies due to normal variations of scapular morphology. This may limit generalised conclusions on shoulder kinematics based on the application of these axes. It also makes comparisons between studies difficult. Kinematics axes that automatically point in the cardinal directions in the anatomic position allow easy application during clinical analysis.

The aim of this study was to quantify axes on clearly identifiable scapular landmarks, to compare these and to compute the best body-fixed coordinate frame that demonstrates the functional cardinal directions and to define 
the landmarks that are least susceptible to scapular morphometric variability.

\section{Materials and Methods}

Twenty-one 3-D image datasets (seventeen CT scans and four cryosectional image sets) covering the complete scapula were used. These were selected retrospectively from various complete scans of the scapula. No scans revealing obvious osseous pathologies including severe arthritis or fragmentations were included. The scanner orientation of the image scans was not considered as a criterion for image selection. Sixteen scans were of left shoulders. The mean age was 60 years (range 57 - 79 years). Nine of the image scans were of $1.0 \mathrm{~mm}$ slice thickness, six $(1.5 \mathrm{~mm})$, four $(1.4 \mathrm{~mm})$ and two $(1.25 \mathrm{~mm})$. There were four contra laterals.

AMIRA image processing software (Mercury Computer Systems Inc, 189 Riverneck Road, Chelmsford, MA, USA) was applied to each image dataset to volumetrically reconstruct the scapula and to extract the 3-D pixel values describing the structures of interest for analysis.

The anatomical directions of an individual scapula that are considered to be close to the cardinal directions were defined. These were the mutually orthogonal anterior, superior and lateral scapular directions with the superior direction being approximately parallel to a bisecting line of the inferior angle. The scapular inferior half is typically triangular in shape and approximating to a thin 'blade' of bony tissue. The plane of the scapular blade was defined using three points (Figure 1). These were the angulus inferior (AI) (Wu et al., 2005), the spine/medial border intersection (SMI) (Wong et al., 2003) and the most inferolateral point of the infra-glenoid tubercle (IGT). A reference medio-lateral axis (Rml) was defined on the plane as a line directed to IGT from SMI. A reference anterior-posterior axis (Rap) was defined as the crossproduct of $\mathrm{Rml}$ and a line from AI to the midpoint of Rml. A mutual orthogonal to Rml and Rap, inferosuperior axis (Ris), was also computed. The three orthogonal axes (Rap, Ris and $\mathrm{Rml}$ ) are specific properties of an individual scapula and are referred to here as 'the blade coordinate frame'. The axes point in the anatomical cardinal directions of the scapula. The blade coordinate frame was used to compute how much a quantified axis deviated from the plane of the scapular body, referred to as the 'axis bearing'.

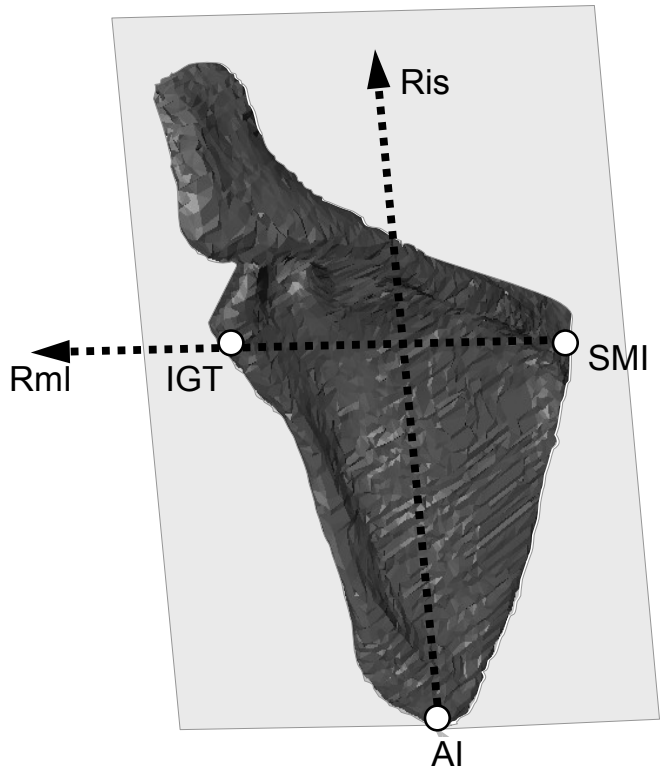

Figure 1: Scapular blade plane
Landmark regions of interest were extracted from each image dataset and reconstructed using a mesh of triangular elements with interconnecting nodes (Figure 2). Each node was a recognisable coordinate in 3-D space. These were saved in individual files. A fourteen-stage protocol was followed for the study of each of the scapulae. This involved the quantification of axes that have been defined previously in the literature and some newly-defined here. These were:

(I) Lateral border line (LBL): The best-fit inferiorsuperior line along the ridge of the scapular lateral border (Hill, 2006). The scapular lateral border is anatomically characterised by a ridge of thickening here referred to as the ridge of the lateral border. Transverse cross-sections of this ridge within the limits of the scapular distal one-third and proximal one-third are nearly elliptical in shape (Figure 3). The centres of the best approximating ellipses to the margins of these cross-sections were mathematically calculated by the segmentation of its outline, reconstruction (Figure 2) and application to geometric shape fitting software. This also quantified the least-square straight line through the centres (Figure 3).

(II) Spine root line (SRL): This describes a long-axis line through the mid substance of the root of the scapular spine. The examination of the cross-section of the spine root, between medial one-third and lateral one-third of an image stack, reveals that this is fundamentally triangular in crosssection in the sagittal plane and nearly straight in the coronal plane (Figure 3). The vertices of the landmark of these crosssections were segmented, reconstructed (Figure 2) and applied to the geometric shape fitting software. This calculated the incentres of the triangles and quantified the least-square straight line through them, directed approximately medio-laterally.

(III) Scapular normal I (SNI): The cross-product (unit vector) between LBL and SRL. By definition, the spine root line and the lateral-border line are located on the body of the scapula and could jointly describe a plane of the scapular body. The cross product between the two would result in an orthogonal to both of them and hence directed approximately anterior posteriorly. The direction cosines of this describe a normal to the plane of the scapula, directed anteriorly.

(IV) Scapular normal II (SNII): The perpendicular line to the least square-plane fit over all the points defining the entire scapular body within the region of distal two-thirds.

(V) Scapular normal III (SNIII): The perpendicular line to the least square-plane fit over all the points defining the rim of the subscapular fossa. In the anterior view, the scapula forms a broad triangle with its medial, lateral and superior borders (Martini et al., 2001; Gosling et al., 1996; Ashton et al., 1965). The borders, up to the suprascapular notch superiorly and infraglenoid tubercle laterally (also called the Superomediolateral Border) form the rim of the concavesubscapular fossa. This was segmented, reconstructed (Figure 2) and applied to the geometric fitting software to quantify the normal unit vector to the plane-fit, directed anteriorly.

(VI) Scapular normal IV (SNIV): The perpendicular line to the least square-plane fit over all the points defining the entire subscapular fossa.

(VII) Scapular normal V (SNV): The perpendicular line to the least square plane fit over all the points defining the anterior aspect of the scapular distal two-thirds (Figure 2).

(VIII) Scapular transverse axis (STA): A line drawn from the mid point of the glenoid fossa to the medial edge on the 
mid glenoid slice as defined by Friedman et al (1992). The axial imaging slices containing the glenoid most inferior and superior aspects, (Zinferior and Zsuperior) were identified and noted. The glenoid middle slice (Zmiddle) was hence calculated as: $Z_{\text {middle }}=Z_{\text {inferior }}+\frac{Z_{\text {superior }}-Z_{\text {inferior }}}{2}$
This was examined and the coordinates representing its anterior, posterior and medial margins were extracted. The midway coordinate between the anterior and posterior margins was calculated and the line joining this from the medial marginal point quantified.
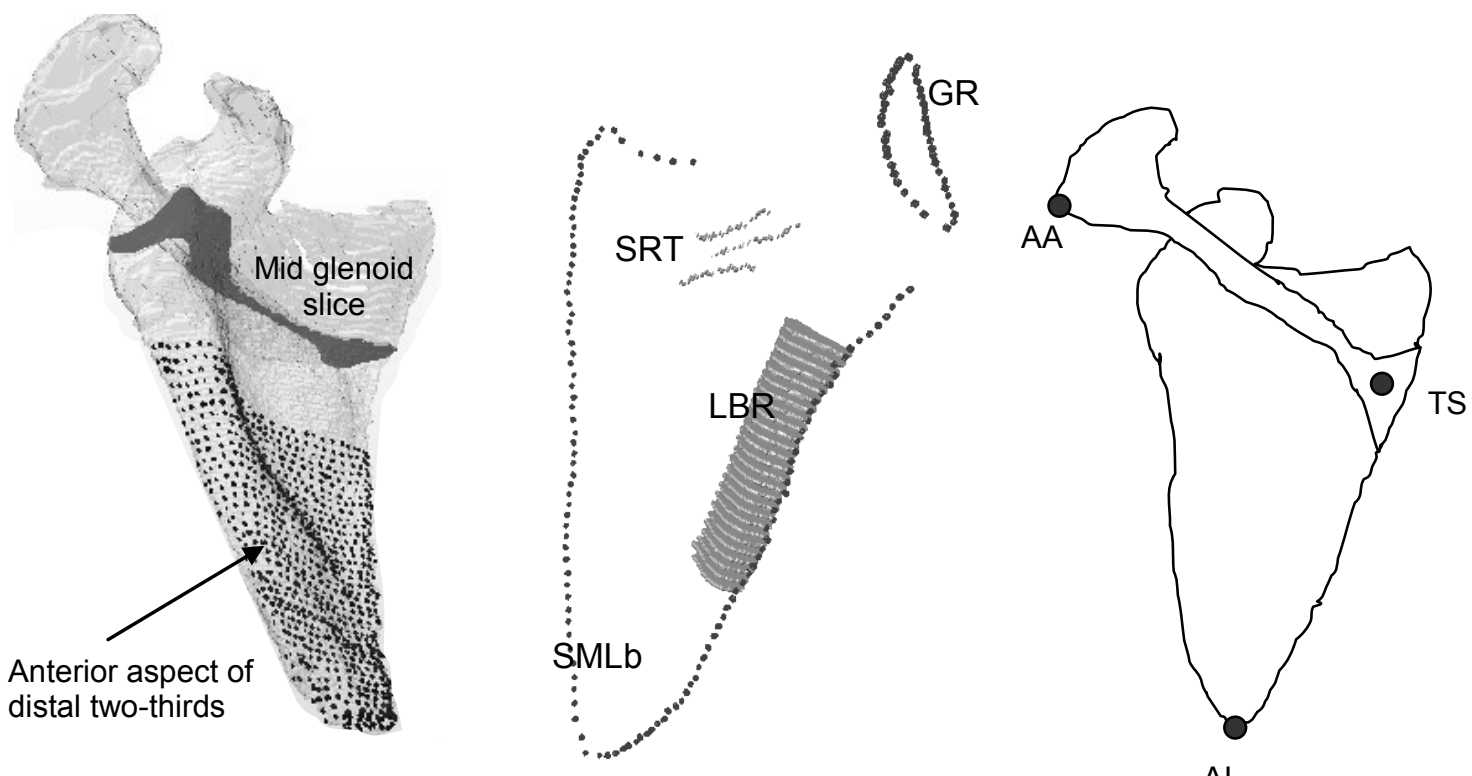

Al

Figure 2: Various extracted scapular regions and landmarks SRT-spine root triangular vertices outline, SMLb-superomedolateral border, LBR-lateral border ridge, GR-glenoid rim

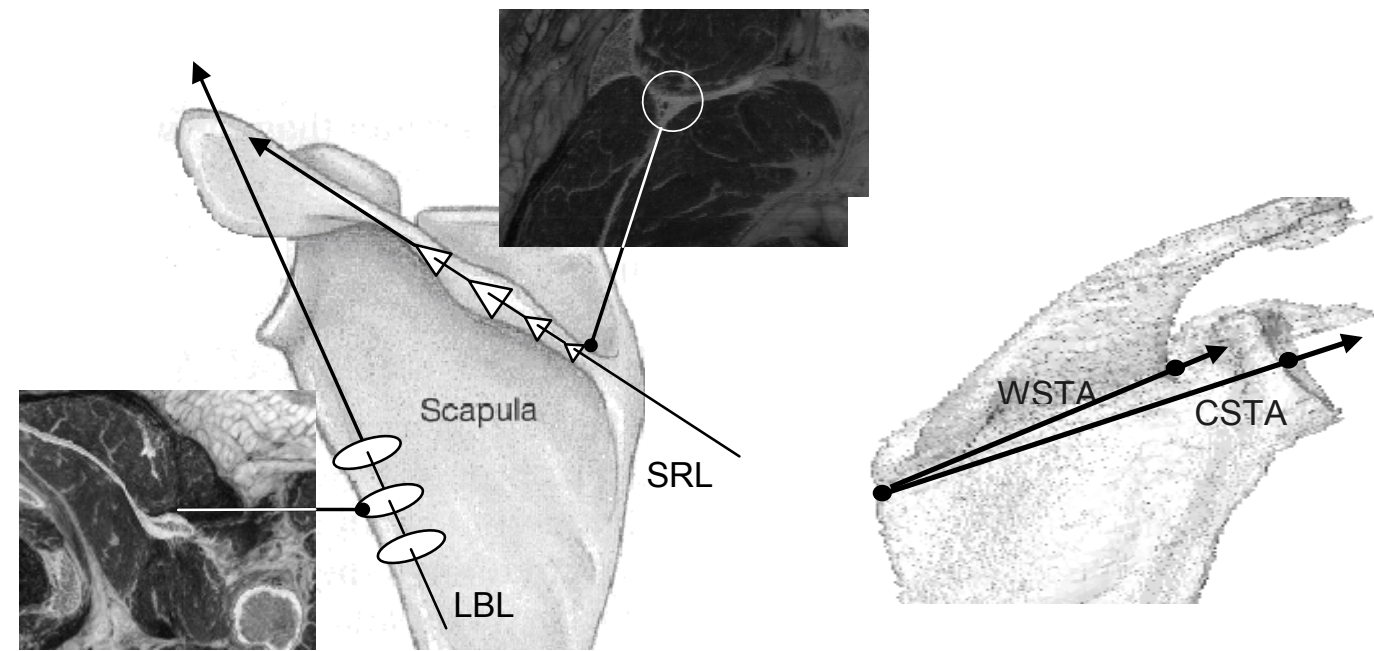

Figure 3: Scapular lateral border (LBL) and spine root (SRL) lines, and Wong (WSTA) and Churchill (CSTA) scapular transverse

\section{axes}

(IX) Bokor scapular transverse axis (BSTA): This was based on the proposals of Bokor et al (1999) that scan orientation should be such that the glenoid surface is perpendicular to the plane of the CT axial cut (Figure 4). The AMIRA obliqueslice tool was applied and the dataset was resliced to be consistent with Bokor et al's proposals. The midglenoid slice of Bokor et al was then identified on the new slices and the BSTA was quantified on it by following the steps described for STA.

(X) Transverse axis of second moment of area (SMATA): The medio-laterally directed principal axis of the 'second moment of area' of the axial slice at the centroid of the glenoid fossa (Figure 2). This definition is concomitant with the so called 'central axis of inertia' of Couteau et al (2000). The glenoid fossa was segmented, reconstructed and applied to calculate the coordinates of its centroid. The nearest axial slice to this was identified, segmented, reconstructed and applied to the geometric shape software. This calculated the medio-laterally directed principal axis of its second moment of area.

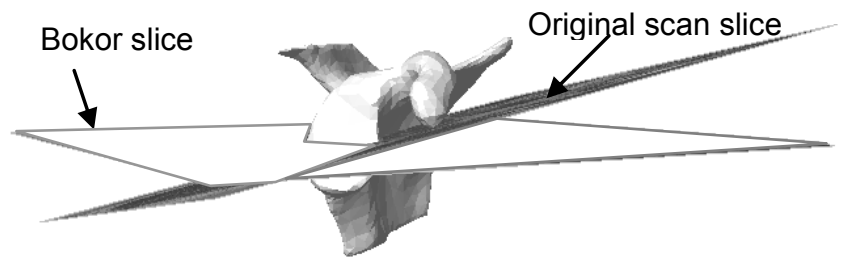

Figure 4: Creating Bokor slices from a previous image scan (Image is re-sliced perpendicular to the glenoid face to obtain Bokor's slices) 
(XI) Wong scapular transverse axis (WSTA): This was a mediolateral line joining the spinoglenoid notch and the spine/medial border intersection (Wong et al., 2003). The 3$\mathrm{D}$ coordinates representing the two points were extracted by the application of AMIRA's pointprobe tool to the reconstructed scapular body and used to quantify the line.

(XII) $\mathrm{Wu}$ medio-lateral axis (WMLA): This was a line joining the 'trigonum spinae scapulae (TS)' and the 'angulus acromialis (AA)' (Wu et al., 2005) (Figure 2). This was quantified following similar steps as in WSTA.

(XIII) $\mathrm{Wu}$ anterior-posterior axis (WAPA): This was the normal to the plane formed by TS, AA and the 'angulus inferior (AI)' (Wu et al., 2005).

(XIV) Churchill scapular transverse axis (CSTA): This represents a line between the centre of the glenoid fossa and the spine/medial border intersection of the scapula (Churchill et al., 2001). The AMIRA pointprobe tool was used to extract the two points as in the case of WSTA.

\section{Statistical Analysis}

Each quantified axis was expressed with respect to the scapular blade coordinate frame and the bearing from it calculated. The bearings from all the specimens for a particular axis were summed and the mean calculated. Axes with mean bearing from the blade coordinate axes greater than $10^{\circ}$ were defined as deviating significantly from the scapular frame of reference. These were eliminated. The remaining axes were subjected to the next elimination round. This considered the insensitivity of the remaining axes to the morphological variations of the scapula. The fourteen axes were quantified from various aspects of the scapular body. These therefore jointly represent the corporate morphology of the scapula. The angles of inclination between each axis and the remainder of the fourteen axes were calculated. The means and standard deviations (SD) of all these angles for all specimens were calculated. A relatively insensitive axis to inter-subject variations in scapular body morphology or pathology would have smaller sum total of its inclination SDs compared to others. The insensitivity index of an axis is therefore defined as the average of its inclination SDs. All the calculated insensitivity indices were divided with smallest index to normalise them relative to the smallest index. The values so obtained were the 'relative insensitivity' of the axes. The final criterion considered was the applicability of an axis even in the presence of pathologies. Axes that are quantified with three or fewer vector points are more likely to lose their integrity in the presence of pathologies. Therefore, risk of failure in pathology was weighted 1 for a 2-point based axis if any of the landmarks associated with its calculation was prone to any regular pathology, otherwise this was 0 . The weighting scores of the last two criteria, insensitivity and applicability, were combined to obtain the final grading of the axes.

At the end of the elimination process, the highest ranking axis was chosen as the first of the three orthogonal axes for the proposed Cartesian coordinate frame (v1). The next top ranking axis on a different anatomical plane from that of $v 1$ was identified as 'v2 temp'. The cross product of v1 and v2temp was calculated. This was orthogonal to both parent vectors and directed to the third anatomical plane. This was the vector, ' $\mathrm{v} 3$ ', the second of the three orthogonal axes of the proposed frame. Finally, the cross product of v1 and v3 produced the last of the three orthogonal axes of the frame, directed approximately in same sense as v2 temp.

\section{Results}

The CSTA of Churchill et al (2001) is the most conforming axis with a bearing of $3.7^{\circ}$ from the $\mathrm{Rml}$ of the scapular blade coordinate frame. Five axes including the WMLA of $\mathrm{Wu}$ et al (2005) had average bearing angle greater than $10^{\circ}$ and were hence eliminated (Table 1). Among the remainder, the CSTA was the most insensitive to variations in scapular body morphology. This scored the smallest value of insensitivity index and was normalised to 1 . The least insensitive axis was the lateral border line with a relative insensitivity of 1.73 (Table 2). In consideration of the volume of quantification points and failure in pathology, CSTA and BSTA weighted 1 while the rest of the axes weighted 0 respectively. In the final grading therefore, the most optimal axis was the spine root line (SRL). This was quantified using many points (Table 2). This was the first of the three orthogonal axes of the scapular kinematics coordinate frame, directed approximately in the medio-lateral sense. The next most optimal axis on a different plane from SRL was the anterior-posteriorly directed SNI. This was hence the second axis for computing the scapular coordinate frame.

\begin{tabular}{|l|l|}
\hline \multicolumn{1}{|c|}{ Axes } & Bearing $\left(^{\circ}\right)$ \\
\hline Churchill Transverse Axis (CSTA) & 3.7 \\
\hline Lateral Border Ridge (LBL) & 4.6 \\
\hline Subscapular Fossa (SNIV) & 4.9 \\
\hline Bokor Transverse Axis (BSTA) & 5.2 \\
\hline Spine Root line (SRL) & 5.7 \\
\hline Wong Transverse Axis (WSTA) & 6.0 \\
\hline Superomediolateral border (SNIII) & 6.6 \\
\hline SRL-LBL Plane normal (SNI) & 8.6 \\
\hline Wu A-P Axis (WAPA) & 9.4 \\
\hline Anterior aspect-inferior 2/3 (SNV) & 12.4 \\
\hline Entire Scapular inferior 2/3 (SNII) & 12.7 \\
\hline Friedman Transverse Axis (STA) & 14.3 \\
\hline Wu M-L Axis (WMLA) & 16.9 \\
\hline $2^{\text {nd }}$ Moment Area (SMATA) & 34.9 \\
\hline
\end{tabular}

Table 1: Relative divergence of axes from scapular blade)

\begin{tabular}{|l|l|l|}
\hline \multicolumn{1}{|c|}{ Axes } & RI & PD \\
\hline Spine Root line (SRL) & 1.05 & 0.00 \\
\hline Wong Transverse Axis (WSTA) & 1.05 & 0.00 \\
\hline Wu A-P Axis (WAPA) & 1.08 & 0.00 \\
\hline SRL-LBL Plane normal (SNI) & 1.18 & 0.00 \\
\hline Superomediolateral border (SNIII) & 1.29 & 0.00 \\
\hline Subscapular Fossa (SNIV) & 1.32 & 0.00 \\
\hline Lateral Border Ridge (LBL) & 1.73 & 0.00 \\
\hline Churchill Transverse Axis (CSTA) & 1.00 & 1.00 \\
\hline Bokor Transverse Axis (BSTA) & 1.08 & 1.00 \\
\hline
\end{tabular}

Table 2: Summary of Relative Insensitivity (RI) and Pathology Dependency (PD) Risk of failure in pathology is 1 for a 2-point based axis if any of the landmarks is associated with any regular pathology, otherwise this is 0 . Relative insensitivity is normalised at 1 for a very high insensitive axis to scapular morphometric variations.

\section{Discussion}

Many current clinical and biomechanical studies that involve scapular motion are based on 2-D axes obtained by surface tracking of landmarks. However, in the context of 3-D modelling of the shoulder, advances in surgical planning and navigation systems, and the ability to conduct advanced imaging of the shoulder, this study focused on obtaining a 
coordinate frame that would be able to be used in advanced kinematic and surgical studies.

Patient specific modelling of the kinematics of the glenohumeral joint requires a robust definition of the bodyfixed Cartesian coordinate frames of the constituent bones. The axes of such a frame need to be close to the standard anatomical directions for easy clinical application without subjective adjustments to achieve alignment. To achieve these requirements and reduce variability especially in the presence of pathologies, the present study applied three criteria to derive the principal axes of the scapular Cartesian coordinate frame. This study has identified the combination of axes to define a body-fixed coordinate frame that is relatively insensitive to inter-scapular variability. The emerged optimal axes (SRL and SNI) have the advantage of being independent of any scanning orientation. They rely on multiple points and are thus less likely to lose their integrity in cases of osseous modifications and fracture (Sugaya et al., 2003). It should be noted that SRL and LBL (the second parent axis of SNI) are quantified from landmarks that are not associated with any common osseous pathology or modifications due to reconstructive surgery. The mediolateral axis defined by Churchill et al (2001) was most insensitive to specimen variability when compared to the other axes. However, this has the disadvantage of depending on only two points and hence might result in large quantification variations in some cases of fracture or osseous modifications. Similarly, Wu et al's (2005) WMLA and WAPA depend on two to three points only but unlike CSTA its landmarks are not commonly affected by any pathology. However, the quantification of these require scans that cover the most proximal and most distal aspects of the scapula. This might require extra radiation exposure since they might not possibly be quantified from standard shoulder or chest scans. It is possible to use magnetic resonance imaging to eliminate radiation exposure, but this would require further work on the accuracy of geometric reconstruction using these images. By definition WMLA points in a postero-lateral direction and this study has demonstrated that this has an average bearing of $16.9^{\circ}$ relative to the plane of the scapular blade. This is a significantly large bearing angle from the lateral direction of the scapula and might pose some difficulties during clinical application of a coordinate frame derived from it. The two emerged optimal axes for the definition of the scapular body-fixed coordinate frame from this study have good indices of insensitivity. Their low bearing angles relative to the plane of the scapula also ensure easy clinical applicability of the coordinate system derived from them. Therefore the derived Cartesian coordinate frame has its three orthogonal axes as (1) a best-fit line through the centre of the spine root, approximately in the medio-lateral direction, (2) the normal to a plane formed by the spine root line and a line through the centre of the ridge of the scapular lateral border, approximately in the anterior-posterior direction, and (3) the crosss-product of axes (1) and (2), directed approximately inferior superiorly. Further studies would apply this as input in modelling patient specific glenohumeral kinematics.

This study has a number of limitations. The main limitation is that 3-D images are required to establish the coordinate frame. This increases the cost of the planned treatment and might introduce ionising radiation to the patient that is not normally included as part of normal clinical practice. An alternative approach to using landmarks would be to use statistical shape modelling or morphing technology to register either a 2-D x-ray or scan, or some digitised surface landmarks to a computational representation of scapular morphology (Yang et al, 2008) Other limitations include the use of landmarks that are not readily accessible, or the arbitrary nature of some of the landmark choices.

In conclusion, this study suggests that when describing scapular kinematics, axes should be chosen based on landmarks that are not likely to be influenced by osseous pathologies. Definition of axes based on two points only should be avoided where possible as this may introduce significant errors. Joint motions are clinically described in terms of rotations and translations along cardinal directions in anatomical position. Therefore, the choice of landmarks for the definition of kinematics axes should be such that the resulting axes do not deviate significantly from these cardinal directions in the anatomical position. This work has proposed one such a definition that is based on the scapular blade plane.

\section{Reference List}

Ashton, E.H., Oxnard, C.E., Spence, T.F., 1965. Scapular shape and primate classification. Proceedings of the Zoological Society, London; 145:125-142.

Bokor, D.J., O’Sullivan, M.D., Hazan, G.J., 1999. Variability of measurement of glenoid version on computed tomography scan. Journal of Shoulder and Elbow Surgery; 8:595 598.

Churchill, R.S., Brems, J.J., Kotschi, H., 2001. Glenoid size, inclination, and version: An anatomic study. Journal of Shoulder and Elbow Surgery; 10:327-332.

Charlton, I.W., 2003. A model for the prediction of the forces at the glenohumeral joint. $\mathrm{PhD}$. thesis, University of Newcastle upon Tyne, United Kingdom.

Couteau, B., Mansat, P., Darmana, R., Mansat, M., Egan, J., 2000. Morphological and mechanical analysis of the glenoid by $3 \mathrm{D}$ geometric reconstruction using computed tomography. Clinical Biomechanics; 15:S8-S12.

Friedman, R.J., Hawthorne, K.B., Genez, B.M., 1992. The use of computerized tomography in the measurement of glenoid version. Journal of Bone and Joint Surgery Am; 74 A:1032 1037.

Grood, E.S., Suntay, W.J., 1983. A joint coordinate system for the Clinical Description of Three Dimensional Motions: Application to the knee. Trans ASME Journal of Biomechanical Engineering; 105:136-144.

Gallino, M., Santamaria, E., Doro, T., 1998. Anthropometry of the scapula: Clinical and surgical considerations. Journal of Shoulder and Elbow Surgery; 7: 284-291.

Gosling, J.A., Harris, P.F., Humpherson, J.R., Whitmore, I., Willan, P.L., 1996. Human Anatomy color atlas and text, Mosby-Wolfe publishers; pp3.30-3.45.

Hill, A.M., 2006. Passive stability of the glenohumeral joint. $\mathrm{PhD}$. thesis, Imperial College, University of London, United Kingdom.

Hill, A.M., Bull, A.M.J., Wallace, A.L., Johnson, G.R., 2007. Qualitative and quantitative descriptions of glenohumeral motion. Gait Posture, article in press, doi:10.1016/j.gaitpost.2007.04.008.

Johnson, G.R., Stuart, P.R., Mitchell, S., 1993. A method for the measurement of three-dimensional scapular movement. Journal of Clinical Biomechanics; 8:269-273 
Martini, F.H., Ober, W.C., Garrison, C.W., Welch, K., Hutchings, R.T., 2001. Fundamentals of Anatomy and Physiology. 5th ed; pp 230-233. Prentice Hall, NJ.

McMahon, P.J., Burkart, A., Musahl, V., Debski, R.E., 2004. Glenohumeral translations are increased after a type II superior labrum anterior-posterior lesion: A cadaveric study of severity of passive stabilizer injury. Journal of Shoulder and Elbow Surgery; 13:39-44.

Monk, A.P., Berry, E., Limb, D., Soames, R.W., 2001. Laser morphometric analysis of the glenoid fossa of the scapula. Clinical Anatomy; 14:320-323.

Novotny, J.E., Beynnon, B.D., Nichols, C.E., 2000. Modeling the stability of the glenohumeral joint during external rotation. Journal of Biomechanics; 33:345-354.

Stehle, J., Moore, S.M., Alaseirlis, D.A., Debski, R.E., McMahon, P.J., 2007. Acromial morphology: Effects of suboptimal radiographs. Journal of Shoulder and Elbow Surgery; 16: 135-142.

Sugaya, H., Moriishi, J., Dohi, M., Kon, Y., Tsuchiya, A., 2003. Glenoid rim morphology in recurrent anterior glenohumeral instability. Journal of Bone and Joint Surgery Am; 85 A:878-884.

Wong, A.S., Gallo, L., Kuhn, J.E., Carpenter, J.E., Hughes, R.E., 2003. The effect of glenoid inclination on superior humeral head migration. Journal of Shoulder and Elbow Surgery; 12:360-364.

Wu, G., van der Helm, F.C.T., Veeger, H.E.J., Makhsous, M., Roy, P.V., Anglin, C., Nagels, J., Karduna, A.R., McQuade, K., Wang, X., Werner, F.W., Buchholz, B., 2005. ISB recommendation on the definitions of joint coordinate systems of various joints for the reporting of human joint motion-Part II:shoulder, elbow, wrist and hand. Journal of Biomechanics; 38:981-992.

Van der Helm, F.C.T., 1997. A standardized protocol for motion recordings of the shoulder. In: Veeger, H.E.J., Van der Helm, F.C.T., Rozing, P.M., (Eds)., Proceedings of the First Conference of the International Shoulder Group; Maastricht, Netherlands.

Van der Helm, F.C.T., 1994. Analysis of the kinematics and dynamic behavior of the shoulder mechanism. Journal of Biomechanics; 27:527-550.

Van der Helm, F., Pronk GM., 1995. Three dimensional recording and description of motions of the shoulder mechanism. Journal of Biomechanical Engineering 117, 27 40.

Yang, Y.H.M., Rueckert, D., Bull, A.M.J., 2008. Predicting the shapes of bones at a joint: application to the shoulder. Computer Methods in Biomechanics and Biomedical Engineering 11, 19-30. 